\title{
Faulty sensor detection using multi-variate sensors in internet of things (IoTs)
}

\author{
Khaldoon Ammar Omar, Ahmed Dhahir Malik, Ansar Jamil, Hasan Muwafeq Gheni \\ Faculty of Electrical and Electronic Engineering, Universiti Tun Hussein Onn Malaysia, Malaysia
}

\section{Article Info}

Article history:

Received Aug 28, 2019

Revised Oct 30, 2019

Accepted Nov 13, 2019

\section{Keywords:}

Faulty detection

IoT

Multivariate sensors

Smart parking

Wireless sensor network

\begin{abstract}
The failure on the IoT devices is also caused due to limited battery life, hardware failure or human mistakes. Sensor faults can be categorized under one type of hardware failure, such as sensor burn, reduced sensor sensitivity and malfunctioned sensors. Any faulty on the IoT devices can cause a problem on the overall operation of the IoT system. Because of this, we proposed a faulty sensor detection and identification mechanism using multivariate sensors. Two methods of decision making are introduced in detecting faulty sensors, which are logical and correlation method that implemented in smart parking system and smart agriculture system accordingly. The logical method compares state of all sensors (ultrasound, IR and hall-effect) in the smart parking system either a parking lot is occupied or available, and then determine the condition of the sensors. The drawback of this method is not able to detect faulty sensor properly for a constant fault, which the sensor reading remains the same value. The correlation method calculates the correlation between all sensors (soil moisture, soil temperature and soil water) in the smart agriculture system. This method uses a moving window technique to calculate the correlation for all sensor over time. Any incomparable and uncorrelated sensor readings means a presence of faulty sensors. Based on the experiment results, the findings shows that the proposed faulty sensor detection mechanism is working properly in detecting faulty sensor in a timely manner.
\end{abstract}

Copyright $(0) 2020$ Institute of Advanced Engineering and Science. All rights reserved.

\section{Corresponding Author:}

A. Jamil,

Wireless and Radio Science Center (WARAS),

Universiti Tun Hussein On Malaysia,

86400 Parit Raja, Batu Pahat, Johor, Malaysia.

Email: ansar@uthm.edu.my

\section{INTRODUCTION}

The concept of a smart city is to merge information and communication technology ICT, and various physical devices connected to the IoT network. Therefore, it optimizes the efficiency of city work, services, increasing population density, mitigate the unexpected challenges and provide a better life for the citizens and visitors [1]. Thus, the word "smart" becomes a cover for ingenious technology which has a degree of artificial intelligence. The main feature of this smart application is the ability to interact with the surrounding environment and get information about the specific area and response accordingly [2]. Therefore, ICT information and communication technology applications such as actuators, sensors and any other devices are necessary means for realizing smart application in any domain [3]. The smart city consists of various smart applications or makes device smart in the city such as smart agriculture, smart parking, smart house, monitoring the climate environment, smart building, smart traffic and transportation systems and etc. $[4,5]$.

For example in smart parking system, the system is developed to deal with the increase in the number of vehicles on the road, people are going to face problems of parking spaces to park their vehicles 
especially in most of the big cities [6]. Drivers spend more time finding and searching for empty parking spaces which causes traffic congestion, consume more fuel, create noise, air pollution and frustration faced by the car drivers [7]. In the world, it is about $30 \%$ of vehicles in the centre of metropolitan cities take about an average of 7.8 minutes searching for empty parking spaces [6]. The annual waste of resource in finding for parking spaces is 47,000 gallons of gasoline consumption, 95,000 hours of time (which about 11 years) and production of 730 tons of $\mathrm{CO} 2$ [7]. In addition, it has been reported that the annual damage to the economy of Schwabing (a district in Germany) to be worth 20 million Euros because of wasting resources in searching parking space [8]. Thus researchers are turned to applying technologies in the management of the parking area by designing and implementation of smart parking systems that helps and allows drivers to effectively find free parking places. The smart parking systems allow drivers to access parking information through their smartphones [9]. This information will help to increase the utilization of spatial parking resource of a city, to reduce unnecessary energy consumption and $\mathrm{CO} 2$ emission of wandering cars, to improve drivers' satisfaction, and to alleviate traffic congestion due to parking guidance itself [10].

After the system design and deployment, it is crucial to detect faults when they occur during the system operational phase [11]. IoT devices are lightweight and have limited computational capabilities often exposed to harsh environments, which can cause failure on the IoT devices [12, 13]. Traditional ways in the management of IoT devices is a maintenance officer require to check each device every day $[12,14]$. Any faulty devices found needs to be fixed or replaced. This traditional method is not practical and very challenging especially in the management of a large scale deployment of IoT consist of hundreds or thousands devices. In light of that, many of the techniques proposed to manage the fault in preventing a sensor network from delivering interruption of service [15]. The fault management technique depends on four relevant as mention: fault prevention, fault detection, fault identification (diagnosis) and isolation and possibly to recover the system from the faulty situation i.e. fault recovery. Different types of fault handling techniques have been implemented by the researchers [16].

\section{PREVIOUS WORKS}

This section provides a review for the existing sensor faulty detection and identification mechanisms. Many efforts has been done to detect and identify faulty sensors in the IoT systems as shown in Table 1.

Table 1. Summary of the Previous Works on Faulty Sensor Detection and Identification

\begin{tabular}{|c|c|c|c|}
\hline \multirow[t]{2}{*}{ Paper } & \multicolumn{2}{|c|}{ Sensor } & \multirow{2}{*}{ Detection } \\
\hline & Single & Multi & \\
\hline Detecting and Identifying Faulty IoT Devices in Smart Home with Context Extraction. [17]. & $\mathrm{X}$ & $\sqrt{ }$ & Cloud \\
\hline A Novel Approach for Faulty Sensor Detection and Data Correction in Wireless Sensor Network [18]. & $\mathrm{X}$ & $\sqrt{ }$ & Node \\
\hline Sensor Data Validation and Abnormal Behavior detection in the Internet of Things [19] & $\mathrm{X}$ & $\sqrt{ }$ & Cloud \\
\hline $\begin{array}{l}\text { Weather Data Analysis and Sensor Fault Detection Using An Extended IoT Framework with Semantics, } \\
\text { Big Data, and Machine Learning [20]. }\end{array}$ & $\mathrm{X}$ & $\sqrt{ }$ & Cloud \\
\hline Trend-adaptive Multi-Scale PCA for data Fault detection in IoT Networks [21]. & $\sqrt{ }$ & $\mathrm{X}$ & Cloud \\
\hline Sensor defect detection in multisensory information fusion [22]. & $\sqrt{ }$ & $\mathrm{X}$ & Node \\
\hline Temporal and spatial correlation based distributed fault detection in wireless sensor networks. [23] & $\sqrt{ }$ & $\mathrm{X}$ & Node \\
\hline Sensor Anomaly Detection in Wireless Sensor Networks for Healthcare. [24] & $\sqrt{ }$ & $\mathrm{X}$ & Node \\
\hline Detection of faulty sensors in IoT using Machine Learning Algorithms. [25] & $\sqrt{ }$ & $\mathrm{X}$ & Cloud \\
\hline
\end{tabular}

The closest work with our paper is proposed by A. Farruggia et al [18], where the faulty sensor detection mechanism using multivariate sensors and the decision making is made on the IoT device itself. They proposed a method based on the spatiotemporal cross-correlation among sensors. The method used two sequential mathematical tools. The first tool is the probabilistic called Markov Random Field MRF to classify the sensor either (work) or (damage). The second stage is learning techniques model, which each sensor basis on its neighbors. In case of sensor working, the model work in the learning stage and sensor is trained, in case of the sensor damaged the corrupt data are replaced with the data generated by the learning model. This mean, the proposed faulty detection mechanism requires learning and training stage using information of each sensor on its neighbors. However, our proposed faulty sensor detection mechanism uses information of multivariate sensor on the same IoT device and do not require learning and training stage. 


\section{METHODOLOGY}

This section describes the design of the proposed faulty sensor detection mechanism using multivariate sensors. The description includes two methods of decision making in detecting faulty sensors: logical and correlation. In addition, this section also explains about the developed prototypes for smart parking system and smart agriculture system.

\subsection{Logical Method of Faulty Sensor Detection in Smart Parking System.}

The proposed smart parking system uses three sensors, which are ultrasonic sensor, IR sensor and hall-effects to sense and detect the presence of car in the parking spaces. The ultrasound sensor is the main sensor used by the system to determine either the parking lot is available or not. If the parking lot is not available, a red coloured LED is switched on and a green coloured LED is switched off. Otherwise, when the parking space is empty, a green coloured LED is switched on and a red coloured LED is switched off. Figure 1 shows the architecture of the developed smart parking system. In term of faulty sensor detection, readings from the three sensors are compared. If all sensors giving a similar reading of occupancy of the parking space, the system is considered working properly and no fault detected. In another hand, if the sensor readings are different, the system detects one or more sensors become faulty. In this condition, both LEDs starts to blink continuously and a notification message is sent to the management office. The decision making to determine condition of each sensor based on the truth table shown in Table 2.

Table 2. Truth Table for Decision Mechanism in Smart Parking

\begin{tabular}{cccc}
\hline Ultrasonic sensor & IR sensor & Hall effect sensor & Decision \\
\hline 0 & 0 & 0 & T (All good) \\
0 & 0 & 1 & F (Hall effect) \\
0 & 1 & 0 & F (IR sensor) \\
0 & 1 & 1 & F (Ultrasonic) \\
1 & 0 & 0 & F (Ultrasonic) \\
1 & 0 & 1 & F (IR sensor) \\
1 & 1 & 0 & F (Hall effect) \\
1 & 1 & 1 & T (All good) \\
\hline
\end{tabular}
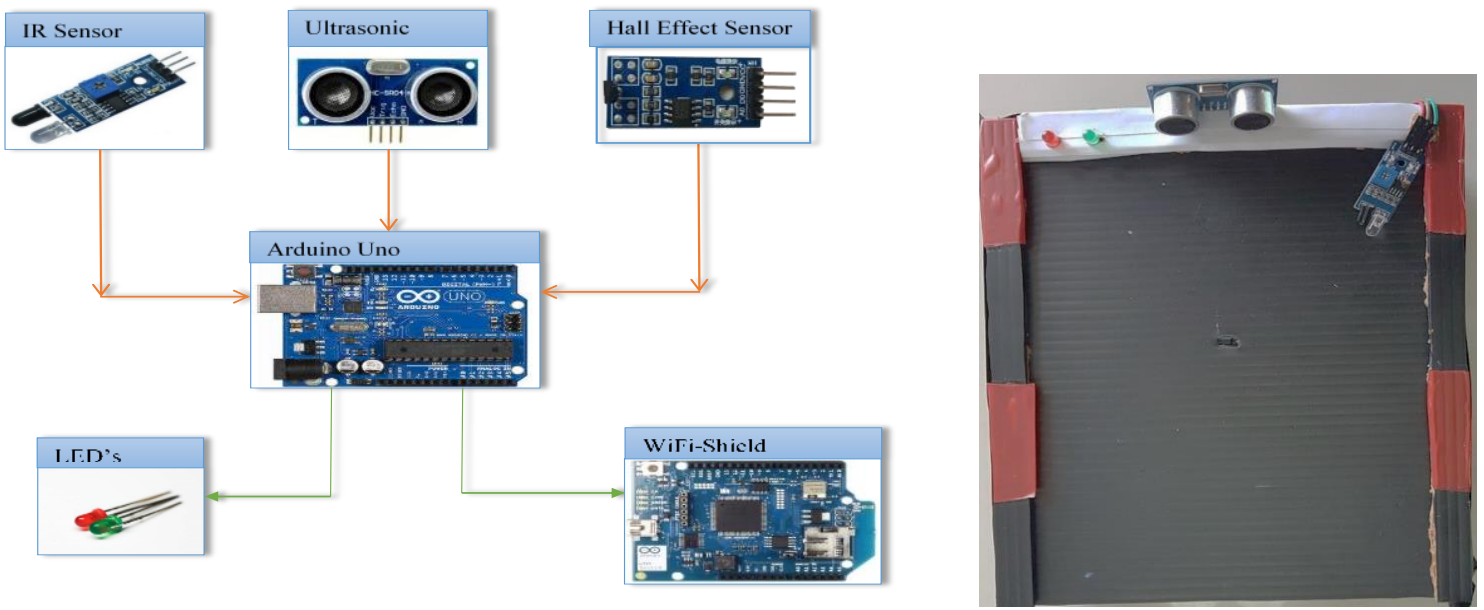

Figure 1. Smart parking system prototype

\subsection{Correlation Method of Faulty Sensor Detection in Smart Agriculture}

The proposed smart agriculture system uses three sensors which are soil moisture (Ms), soil water (Ws) and soil temperature (Ts) to monitor health of plants continuously. The system collects sensor reading for every 30s and send the data to the management office via internet. Figure 2 shows architecture of the developed smart agriculture prototype. This method uses correlation between readings of these three sensors to determine any faulty sensor. It is very important for all sensor readings to have strong correlations in order for this method to work accurately. In this smart agriculture system, soil moisture readings and soil water readings have a positive correlation. The soil moisture and soil temperature readings have a negative correlation. The soil water and soil temperature sensor readings have a negative correlation. Any uncorrelated sensor readings is an indication of faulty sensors. 
The decision mechanism to determine condition of each sensor based on the relationship of correlation between these three sensors as illustrated in Table 3. Based on table, there are four decisions can be made which are good, faulty_Ms, faulty_Ts, faulty_Ws, faulty_all_sensors and don't_care. Good decision means all sensors in good condition and working perfectly. Then, faulty_Ms, faulty_Ts, and faulty_Ws mean faulty soil moisture, faulty soil temperature and faulty soil water sensor accordingly. Faulty all sensors means all sensors in faulty condition. Faulty two sensors decision is considered two or more sensors are faulty. For example the first combination, correlation of moisture sensor and water sensor (SM-SW) is false and the other two correlations are correct. When (SM-SW) is uncorrelated, it shows that one of the sensor become faulty either moisture or water sensor. However, correlation of both sensors with soil temperature are correct indicating both soil moisture and soil water in good condition, which is a situation that impossible to happen for one sensor become faulty. Because of this, the first combination is considered as Faulty two sensors decision.

The calculation of correlations between the three sensors are based on moving window technique. The window size determines number of sensor data that is considered during calculation of correlation. The larger number of sensor data provide a better correlation to represent the actual experiment. This window is moved along the time depending on the moving window time parameter. In this experiment, the window size is set to 5 minutes (300s), which 10 sensor data are considered in the calculation on correlations. This mean, the moving window time will be 5 minutes (300s).

Table 3. Decision based on Correlation between Three Sensor Readings in Smart Agriculture

\begin{tabular}{|c|c|c|c|}
\hline ST-SM & ST-SW & SM-SW & Decision \\
\hline [-ve, correct] & [-ve, correct] & {$[$ [-ve, false] } & Faulty two sensors \\
\hline$[-\mathrm{ve}$, correct $]$ & {$[-\mathrm{ve}$, correct $]$} & [+ve, correct] & Good \\
\hline$[-\mathrm{ve}$, correct $]$ & [+ve, false] & {$[-\mathrm{ve}$, false $]$} & Faulty_Ws \\
\hline [-ve, correct] & [+ve, false] & [+ve, correct] & Faulty two sensors \\
\hline$[+\mathrm{ve}$, false] & [-ve, correct] & {$[-\mathrm{ve}$, false] } & Faulty_Ms \\
\hline [+ve, false] & {$[-\mathrm{ve}$, correct $]$} & [+ve, correct] & Faulty two sensors \\
\hline$[+\mathrm{ve}$, false $]$ & [+ve, false] & {$[-v e$, false $]$} & Faulty all sensors \\
\hline [+ve, false] & [+ve, false $]$ & [+ve, correct] & Faulty_Ts \\
\hline
\end{tabular}
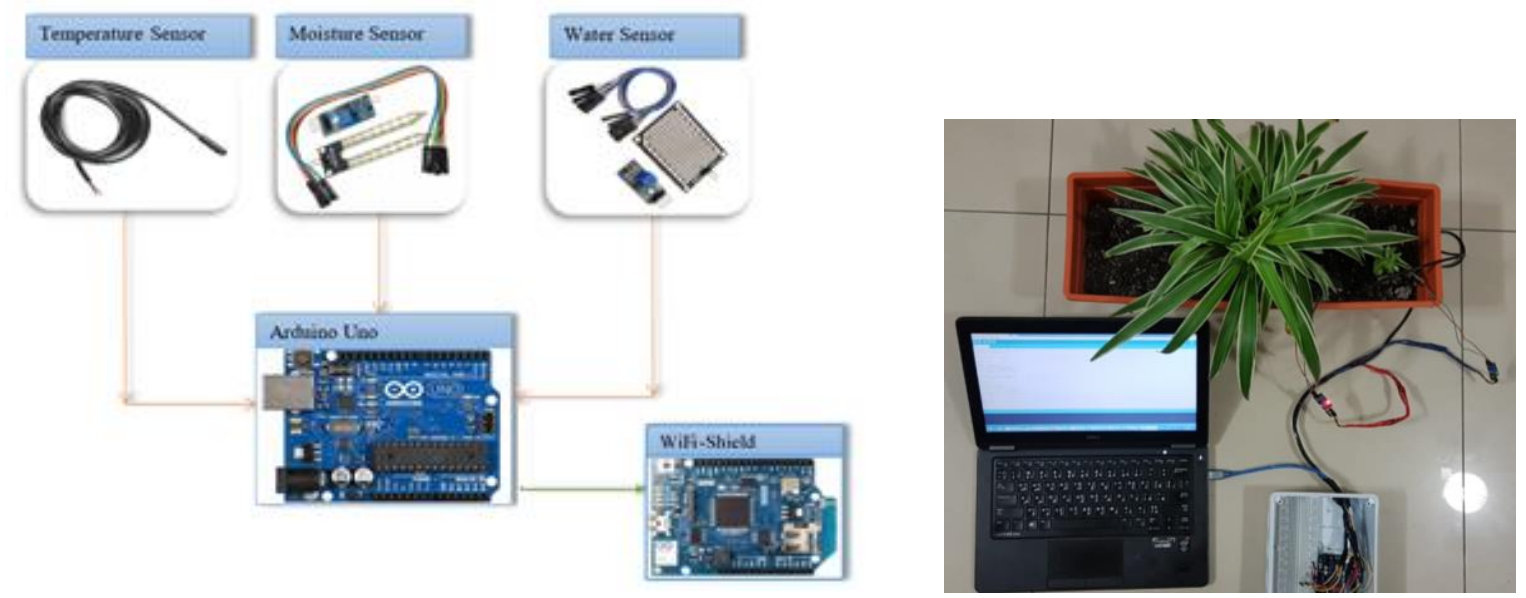

Figure 2. Smart agriculture prototype

\section{RESULTS}

In this section, the result and analysis for the proposed faulty sensor detection mechanism are discussed. These analysis include for both methods of faulty sensor detection. Several experiments were conducted to determine the performance of the proposed faulty sensor detection mechanism in term of its functionality and detection of faulty sensor. A smart parking prototype was built to test the logical method of faulty sensor detection. The smart parking system detects availability status of parking lot either is empty or occupied. In order to test the second method, a smart agriculture prototype was built to monitor soil conditions: soil moisture, soil water and soil temperature.

One of the smart parking experiments was conducted by having the initial 5 seconds for an empty parking and the next 5 seconds with occupied parking space. Then, it repeated for 10s with empty packing 
space and the next 10 s with occupied parking space. The results show that all sensors giving similar readings as expected that indicate all sensors in good condition as shown in Figure 3. The green LED switched on when the parking is empty and the red LED is turned on when the parking is occupied.
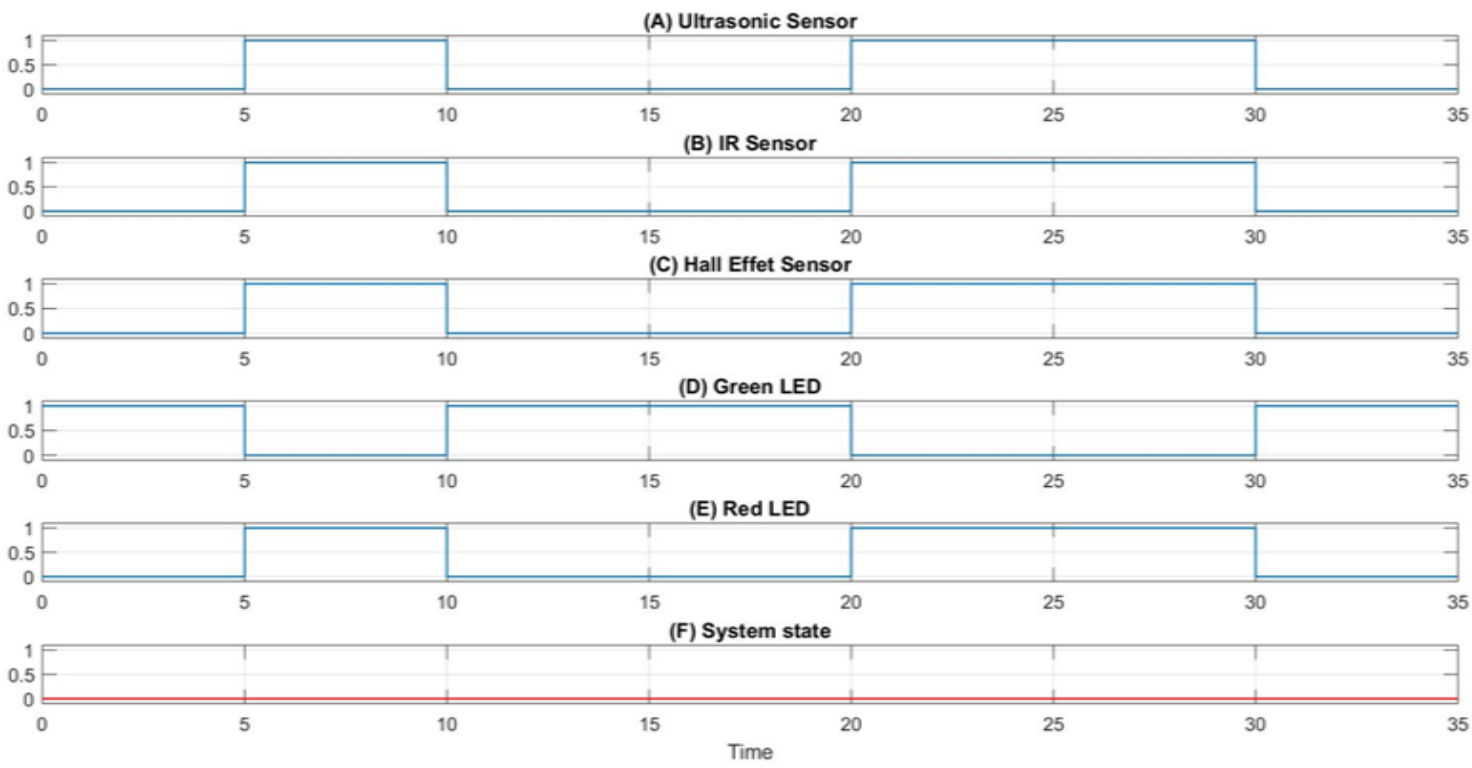

Figure 3. State of three sensors in the system all sensors in good condition

Figure 4 shows the experimental result for one sensor in a faulty condition and the other two sensors in good condition. The faulty sensor is the hall-effect sensor that gives different readings from the other two sensors. This experiment simulates a situation where two sensors detect the state of parking space correctly and one sensor detects wrongly. Based on the results, the sensor faulty detection mechanism shows that the system is considered to be at faulty when the hall-effect sensor started to give the wrong reading at $5 \mathrm{~s}$. At the same time, both LEDs start to blink as shown in (E) and (F). The most important thing, the faulty mechanism can determine that the hall-sensor is the faulty sensor based on the truth table.
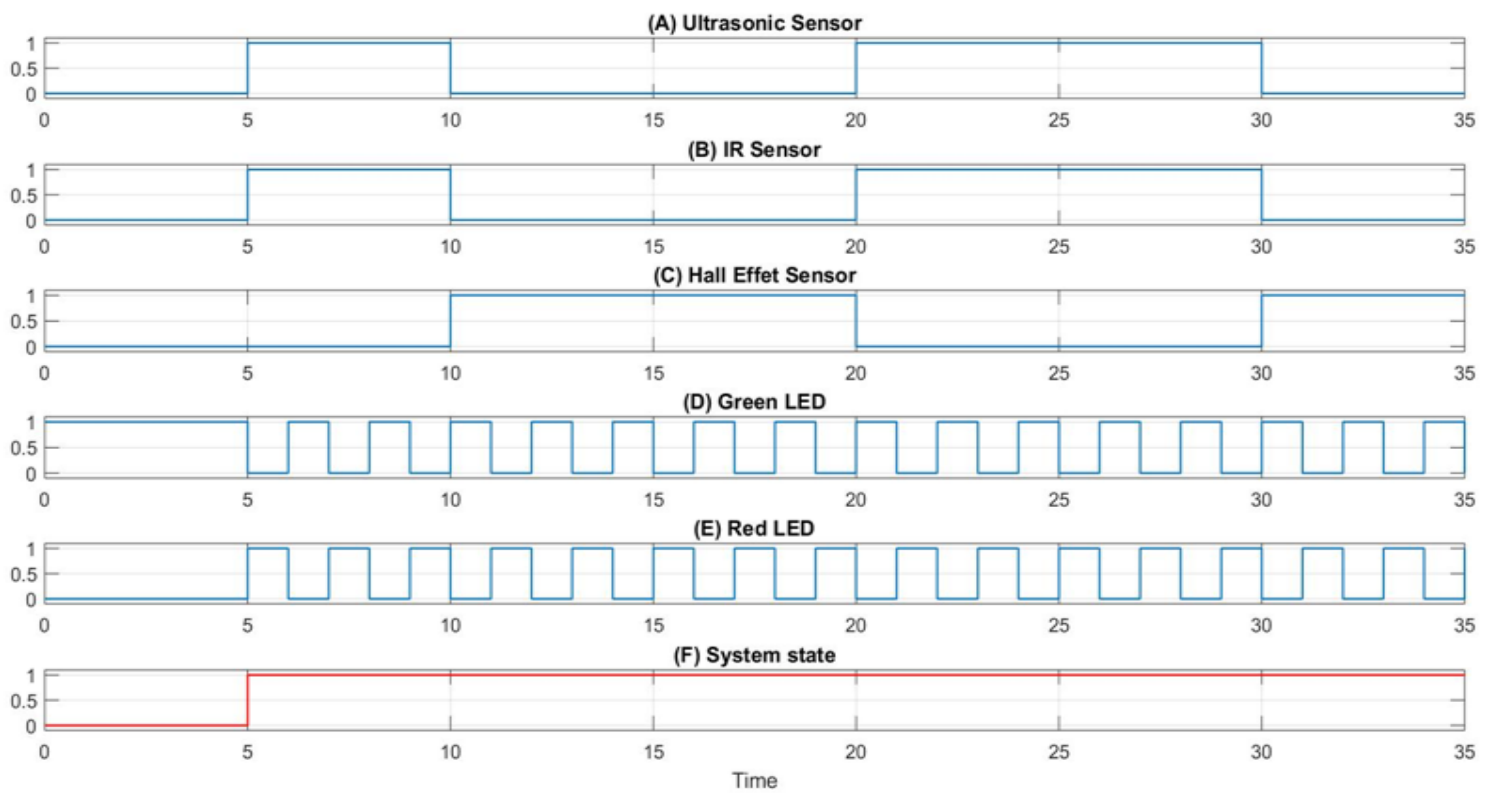

Figure 4. State of three sensors in the system for one sensor in a faulty condition 
Figure 5 shows the experimental results for when one sensor giving a constant fault with a low state reading. In this experiment, we consider the IR sensor in a faulty condition which always giving a constant zero reading. The other two sensors are working properly. Based on the result, it is found that the faulty detection mechanism able to detect the faulty sensor (using the truth table) when the parking space is occupied. Otherwise, when the parking space is empty, the faulty mechanism failed to detect the faulty sensor.

In the correlation method of faulty detection, experiments are conducted using smart agriculture prototype, which provide information of soil conditions: soil moisture, soil water and soil temperature. The system aims to sense the information of the soil and predicting the fault when that happens. In the experiments, water was dripped in the pot gradually to water the plant for about 35 minutes. The collected sensor readings is plotted into a graph as shown in Figure 6. Based on the graph, the soil water reading increases from $64 \%$ to $88 \%$. The soil moisture sensor increases from $43 \%$ to $92 \%$. Otherwise, the temperature drops from $29.71^{\circ} \mathrm{C}$ to $26.61^{\circ} \mathrm{C}$, which is about $0.1 \%$ reduction. The finding shows that the soil water and soil moisture has a strong correlation but both sensors has a weak correlation with the soil temperature sensor. The faulty sensor detection mechanism shows all sensors are in good condition as expected. The decision made by the mechanism shown in Table 4. ST-SM means correlation between soil moisture and soil moisture. ST-SW means correlation between soil temperature and soil water. SW-SM means correlation between soil water and soil-moisture.
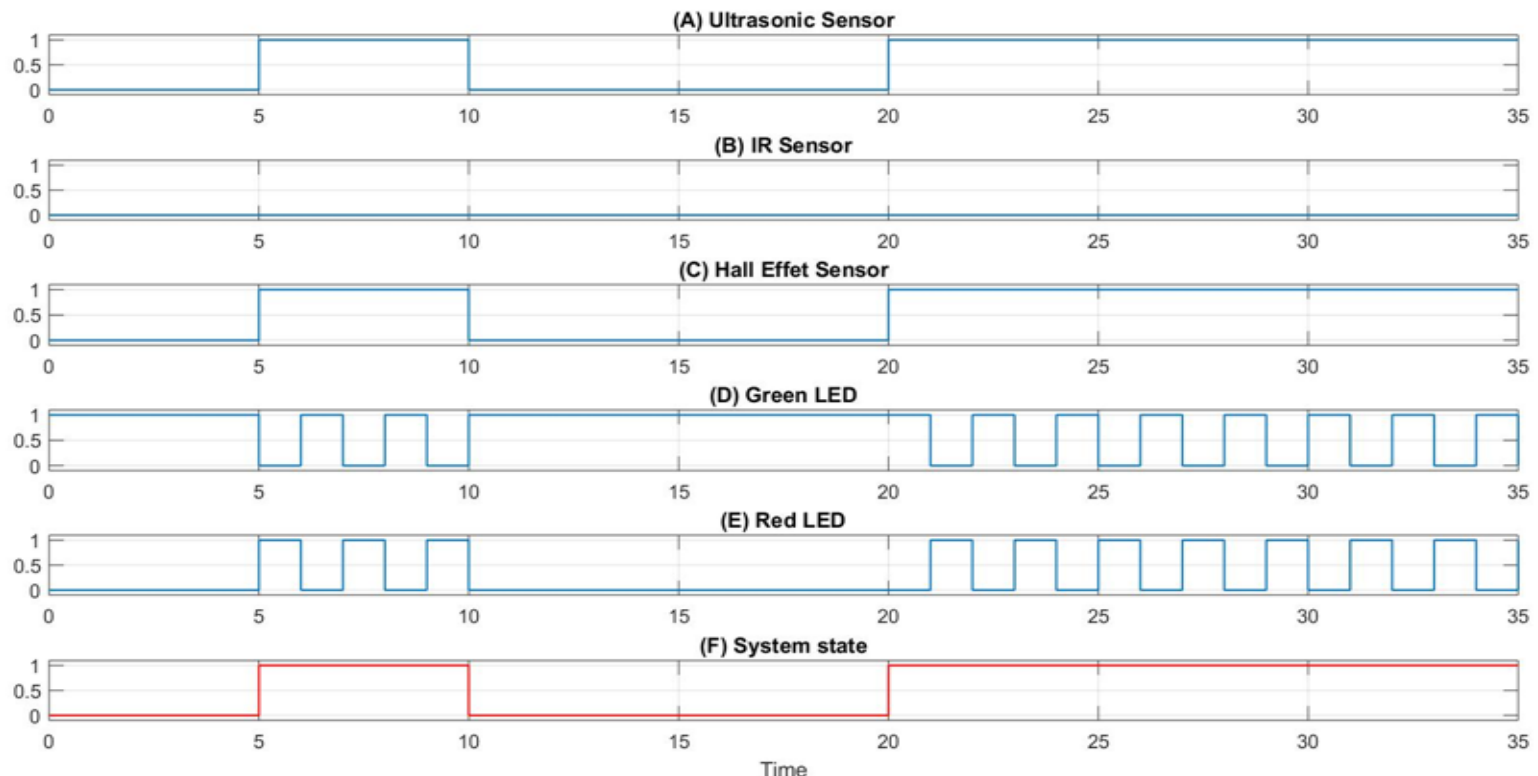

Figure 5. State of three sensors in the system when one sensor giving a constant fault

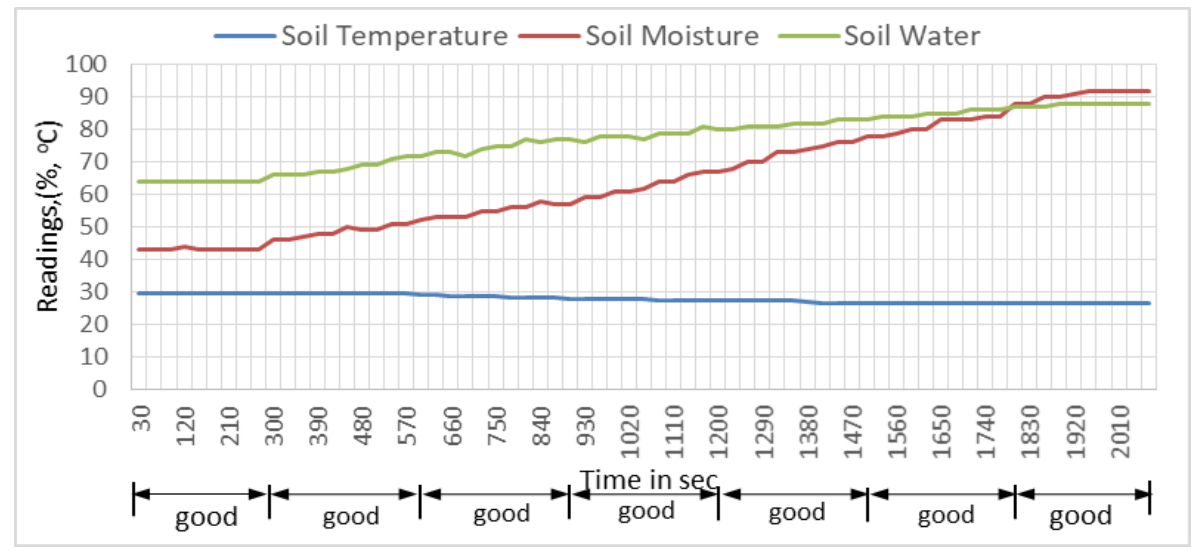

Figure 6. Sensor readings data for smart agriculture 
As the comparison, we manipulated the sensor data to introduce a faulty sensors. The water sensor data was modified at time from 20 minutes and onward as shown in Figure 7. Instead of having an increase trend of the original sensor data, the modified data has a reduce trend. Based on the graph, the mechanism able to detect faulty soil water sensor after calculating correlations for moving time of 20 minutes. This means, the mechanism detects the faulty at 25 minutes, which is 5 minutes after the sensor become faulty. Table 4 shows that correlation of ST-SW and SW-SM gives wrong correlation values. Correlation of ST-SW has changed from negative correlation to positive correlation. Otherwise, correlation of ST-SM has changed from positive correlation to negative correlation. However, correlation of ST-SM maintains to be negative correlation, which it is the correct correlation. In this case, the mechanism decides that soil water is at faulty condition.

Table 4. Condition Status of Sensor for Original and Manipulated Data in the Smart Agriculture

\begin{tabular}{ccccccccc}
\hline \multirow{2}{*}{ Moving time (Minutes) } & \multicolumn{4}{c}{ Original data } & \multicolumn{4}{c}{ Manipulated data } \\
& ST-SM & ST-SW & SW-SM & Sensor status & ST-SM & ST-SW & SW-SM & Sensor status \\
\hline 5 & -0.600 & -0.667 & -0.600 & Good & -0.600 & -0.667 & 0.946 & Good \\
10 & -0.759 & -0.817 & -0.759 & Good & -0.759 & -0.817 & 0.938 & Good \\
15 & -0.898 & -0.889 & -0.898 & Good & -0.898 & -0.889 & 0.895 & Good \\
20 & -0.941 & -0.855 & -0.941 & Good & -0.941 & -0.855 & 0.862 & Good \\
25 & -0.886 & -0.886 & -0.886 & Good & -0.874 & 0.716 & -0.892 & FaultyWs \\
30 & -0.933 & -0.924 & -0.933 & Good & -0.933 & 0.701 & -0.656 & Faulty Ws \\
35 & -0.824 & -0.662 & -0.824 & Good & -0.824 & 0.709 & -0.453 & Faulty Ws \\
\hline
\end{tabular}

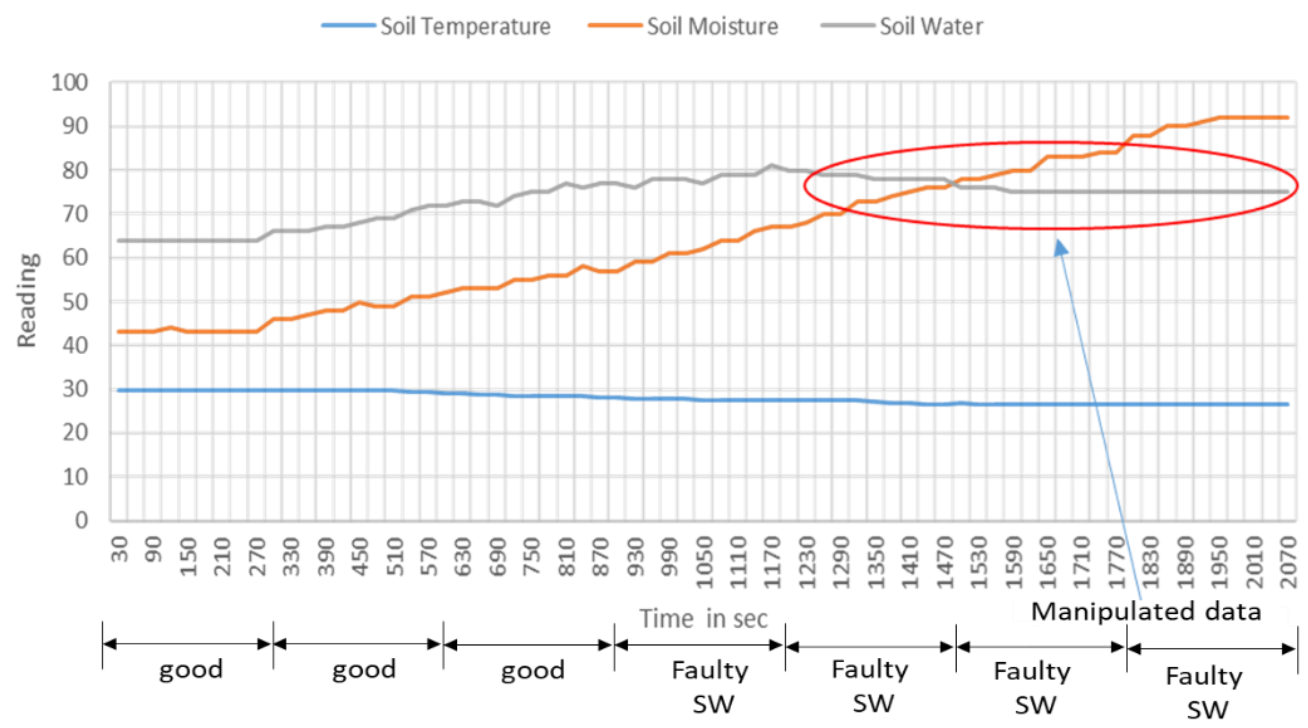

Figure 7. Manipulated sensor data for smart agriculture

\section{CONCLUSION}

In this project, a faulty sensor detection and identification mechanism using multivariate sensors is designed successfully. In order to decide faulty sensor, the mechanism uses two methods: logical and correlation method. The logical method decision making is implemented in the developed prototype of smart parking system. This method uses three different types of sensor, which are ultrasound sensor, IR sensor and hall-effect sensor. The ultrasound sensor is used as the main sensor in detecting either a parking space is available or not. The other two sensors are used for faulty sensor detection mechanism. The mechanism compares all sensor readings to detect any faulty sensor. If all sensors giving a similar reading of availability of the parking space, the system is working properly and no fault detected. In another hand, if the sensor readings are different, the faulty sensor is detected. The mechanism is found to have one drawback that not able to detect properly a fault sensor that provides a constant reading. In the correlation method, the decision making in detecting faulty sensor by finding correlation between sensors. This method is implemented in the prototype of smart agriculture system to monitor health of plants continuously that attached with three type of sensors: soil moisture, soil water and soil temperature. Soil moisture and soil temperature readings have a negative correlation. Soil water and soil temperature sensor readings have a negative correlation. Otherwise,

Faulty sensor detection using multi-variate sensors in internet of things (IoTs) (Khaldoon Ammar Omar) 
Soil moisture readings and soil water readings have a positive correlation. Any uncorrelated sensor readings is an indication of faulty sensors. The calculation of correlations between the three sensors are based on moving window technique. The window size determines number of sensor data considered during calculation of correlation. The larger number of number of sensor data will provide a better correlation to represent the actual experiment. This window is moved along the time based on the moving window time parameter.

\section{ACKNOWLEDGEMENTS}

The authors would like to express their thanks to Universiti Tun Hussein Onn Malaysia (UTHM) for providing financial support under H132-TIER 1 Grant.

\section{REFERENCES}

[1] H. Chourabi et al., "Understanding smart cities: An integrative framework," Proc. Annu. Hawaii Int. Conf. Syst. Sci., pp. 2289-2297, 2012.

[2] D. Marikyan, S. Papagiannidis, and E. Alamanos, "A systematic review of the smart home literature: A user perspective," Technol. Forecast. Soc. Change, vol. 138, no. November 2017, pp. 139-154, 2019.

[3] C. T. Yin, Z. Xiong, H. Chen, J. Y. Wang, D. Cooper, and B. David, "A literature survey on smart cities," Sci. China Inf. Sci., vol. 58, no. 10, pp. 1-18, 2015.

[4] T. Nam and T. A. Pardo, "Proceedings of the 12th Annual International Digital Government Research Conference: Digital Government Innovation in Challenging Times," Coll. Park. Maryland, USA, 2011, pp. 282-291, 2011.

[5] S. A. Chelloug and M. A. El-Zawawy, "Middleware for Internet of Things: Survey and Challenges," Intell. Autom. Soft Comput., vol. 44006, no. February 2016, pp. 1-9, 2017.

[6] Q. G. K. Safi, S. Luo, L. Pan, W. Liu, R. Hussain, and S. H. Bouk, "SVPS: Cloud-based smart vehicle parking system over ubiquitous VANETs," Comput. Networks, vol. 138, pp. 18-30, 2018.

[7] K. Hassoune, W. Dachry, F. Moutaouakkil, and H. Medromi, "Smart parking systems: A survey," SITA 2016 11th Int. Conf. Intell. Syst. Theor. Appl., 2016.

[8] J. H. Shin and H. B. Jun, "A study on smart parking guidance algorithm," Transp. Res. Part C Emerg. Technol., vol. 44, pp. 299-317, 2014.

[9] S. L. E.H. Wu, C. Liu, J. Sahoo, M. Jin, “Agile Urban Parking Recommendation Service for Intelligent Vehicular Guiding System," N Engl Bioeng Conf, pp. 35-49, 2014.

[10] D. C. Shoup, “Cruising for parking,” Transp. Policy, vol. 13, no. 6, pp. 479-486, 2006.

[11] E. U. Warriach, M. Aiello, and K. Tei, "A Machine Learning Approach for Identifying and Classifying Faults in Wireless Sensor Network,” 2012 IEEE 15th Int. Conf. Comput. Sci. Eng., pp. 618-625, 2012.

[12] T. W. Hnat et al., "The hitchhiker's guide to successful residential sensing deployments," Proc. 9th ACM Conf. Embed. Networked Sens. Syst. - SenSys '11, no. May, p. 232, 2011.

[13] T. Kavitha and D. Sridharan, "Security Vulnerabilities In Wireless Sensor Networks: A Survey," J. Inf. Assur. Secur., vol. 5, no. October, pp. 31-44, 2010.

[14] K. Kapitanova, E. Hoque, J. A. Stankovic, K. Whitehouse, and S. H. Son, "Being SMART about failures: assessing repairs in SMART homes," Ubicomp 2012, pp. 51-60, 2012.

[15] D. Raposo, A. Rodrigues, J. S. Silva, and F. Boavida, "A Taxonomy of Faults for Wireless Sensor Networks," J. Netw. Syst. Manag., vol. 25, no. 3, pp. 591-611, 2017.

[16] S. Sciences, "A systematic literature review in fault analysis for IoT Guru Prasad Bhandari * Ratneshwer Gupta," vol. X, pp. 1-18, 2018.

[17] J. Choi et al., "Detecting and identifying faulty IoT devices in smart home with context extraction," Proc. - 48th Annu. IEEE/IFIP Int. Conf. Dependable Syst. Networks, DSN 2018, pp. 610-621, 2018.

[18] A. Farruggia and S. Vitabile, "A Novel Approach for Faulty Sensor Detection and Data Correction in Wireless Sensor Network," 2013 Eighth International Conference on Broadband and Wireless Computing, Communication and Applications, Compiegne, 2013, pp. 36-42.

[19] H. Sándor, B. Genge and Z. Szántó, "Sensor data validation and abnormal behavior detection in the Internet of Things," 2017 16th RoEduNet Conference: Networking in Education and Research (RoEduNet), Targu Mures, 2017, pp. 1-5.

[20] A. C. Onal, O. Berat Sezer, M. Ozbayoglu and E. Dogdu, "Weather data analysis and sensor fault detection using an extended IoT framework with semantics, big data, and machine learning," 2017 IEEE International Conference on Big Data (Big Data), Boston, MA, 2017, pp. 2037-2046.

[21] T. Dang, M. Tran, D. Le, V. V. Zalyubovskiy, H. Ahn and H. Choo, "Trend-adaptive multi-scale PCA for data fault detection in IoT networks," 2018 International Conference on Information Networking (ICOIN), Chiang Mai, 2018, pp. 744-749.

[22] Ehlenbröker, J.-F., Mönks, U., and Lohweg, V. "Sensor defect detection in multisensor information fusion", J. Sens. Sens. Syst., 5, 337-353.

[23] Yu, A. M. Akhtar, X. Wang and A. Shami, "Temporal and spatial correlation based distributed fault detection in wireless sensor networks," 2015 IEEE 28th Canadian Conference on Electrical and Computer Engineering (CCECE), Halifax, NS, 2015, pp. 1351-1355. 
[24] Haque SA, Rahman M, Aziz SM. "Sensor anomaly detection in wireless sensor networks for healthcare". Sensors (Basel). 2015;15(4):8764-8786.

[25] Adikari, Hiran. "Detection of faulty sensors in IoT using Machine Learning Algorithms", (2017).

\section{BIOGRAPHIES OF AUTHOR}
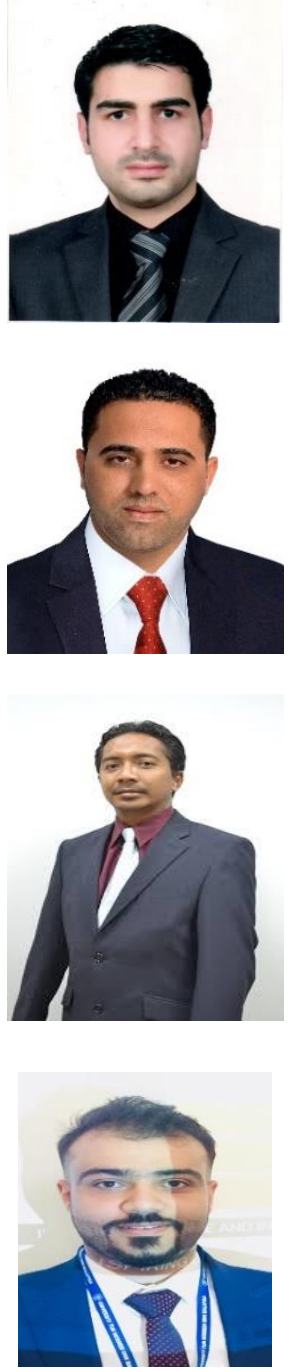

Khaldoon Ammar omar, was born in Mosul-Iraq, on January 10, 1991. Graduated from Mosul University in Electronics Engineering in the year 2012. Now is getting his MSc degree in Electronics and Communication Engineering from UTHM University in the year 2019.

Ahmed Dhahir Malik was born in Iraq, on May 12, 1984.graduated from University of Iraq in communication system Engineering in the year 2011. He has submitted his master thesis at IOTs in UTHM Malaysia. Presently, he is working as engineer in IT.

Ansar Jamil is currently a lecturer in the department of Communication Engineering, Faculty of Electric and Electronic Engineering, UTHM. He is also an active researcher in Wireless and Radio Science (WARAS) center. He received B.Eng and M.Eng. in Electronics and Telecommunications from Universiti Teknologi Malaysia (UTM), Malaysia in 2005 and 2009 respectively. He gained his $\mathrm{PhD}$ in Electronic, Electrical and Systems Engineering from Loughborough University in 2015. He has two years experiences as an Electronic Engineer in RnD Department of Motorola, before joining UTHM as tutor for 8 years. His main areas of research interest are wireless sensor network and IoT

Hasan Muwafeq Gheni, was born in Iraq, on January 2, 1995. Graduated from University of Babylon in Electrical Engineering in the year 2015. He has submitted his master thesis at IOTs in UTHM Malaysia. 